\title{
Effect of gold nanoparticle solution on VDRL reactive CSF
}

\author{
Efeito da solução de nanopartículas de ouro na reação de VDRL reagente no LCR
}

Sir, the application of the novel nanotechnology in diagnostic medicine is very interesting. In neurology, there are some previous reports. Focusing on the cerebrospinal fluid (CSF) examination, some previous publications previously mentioned for the application of gold nanoparticle solution as a diagnostic reagent for protein and Cryptococcus determination within $\mathrm{CSF}^{1,2}$. Here, the author additionally reports the observation on testing effect of gold nanoparticle on VDRL reactive CSF. The gold nanoparticle solution was prepared as previously described in referencing papers ${ }^{1,2}$ and the test system was between prepared solution and CSF sample. Overall $20 \mathrm{CSF}$ samples, $10 \mathrm{VDRL}$ reactive and 10 non VDRL reactive were included into the test system. Of interest, in this preliminary observation, all non VDRL reactive samples remained unchanged (grey color of added solution) when reacted with test solution whereas all VDRL reactive samples show color change (from grey to red). In fact, there are some recent reports confirming the usefulness of gold nanoparticle techniques for help study syphilis. The good example is the report by Yang et al on Polyelectrolyte coated gold magnetic nanoparticles as a point of care testing system for determination of syphilis ${ }^{3}$. However, it does not directly focus on neurosyphilis ${ }^{3}$. Here, it can be seen that there is a nano-chemical reaction occurring within the VDRL reactive sample. This might imply the possible application of gold nanoparticle solution as a naked eye screening system. However, to confirm this observation, a larger systematic evaluation is needed.

Viroj Wiwanitkit ${ }^{1,2,3,4}$

\section{References}

1. Wiwanitkit V. Gold nanoparticle as an alternative diagnostic solution for detection protein in cerebrospinal fluid. J Neurol Sci 2010;27:383.

2. Sereemaspun A, Wiwanitkit V, Rojanathanes R. Interaction between gold nanoparticles and cryptococcus spp. Arch Hellenic Med 2009;26:520-522.
3

Yang D, Ma J, Zhang Q, et al. Polyelectrolyte coated gold magnetic nanoparticlesfor immunoassay development: toward point of care Diagnostics for syphilis screening. Anal Chem 2013;85:6688-6695.

${ }^{1}$ Hainan Medical University, China;

${ }^{2}$ Faculty of Medicine, University of Nis, Serbia;

${ }^{3}$ Joseph Ayobabalola University, Nigeria,

${ }^{4}$ Chulalongkorn University, Bangkok Thailand

Correspondence: Viroj Wiwanitkit; Wiwanitkit House, Bangkhae, Bangkok Thailand; Email: wviroj@yahoo.com

Conflict of interest: There is no conflict of interest to declare.

Received 25 November 2013; Received in final form 13 December 2013; Accepted 03 January 2014. 